\title{
¿Son las fincas agroecológicas resilientes? Algunos resultados utilizando la herramienta SHARP-FAO en Costa Rica
}

\author{
Are Agroecological Farms Resilient? Some Results Using the Tool \\ SHARP-FAO in Costa Rica
}

\author{
Sergio A. Molina-Murillo \\ Univesidad Nacional de Costa Rica (UNA), \\ Univesidad de Costa Rica (UCR) \\ sergiomolina@una.cr \\ Guido Barrientos, Maximiliano Bonilla, Cristian Garita, Alejandro Jiménez, \\ Mairon Madriz, Javier Paniagua, Juan Carlos Rodríguez, Laura Rodríguez, \\ Jairo Treviño, Sandra Valdés \\ Estudiantes de Maestría en Agricultura Ecológica, \\ Universidad Nacional de Costa Rica (UNA)
}

Recibido: 31 de enero 2017

Aceptado: 10 de marzo 2017

\begin{abstract}
Resumen
Medir y monitorear la resiliencia de los sistemas agrosilvopastoriles de pequeños productores es cada vez más urgente para ayudarles a enfrentar de mejor manera los efectos del clima cambiante. Aunque se reconocen otras formas de medir la resiliencia climática, la novedosa herramienta SHARP desarrollada por la FAO permite su identificación, medición y la priorización de acciones para mejorarse de una manera participativa, tomando en cuenta los intereses de los productores. En coordinación con el equipo desarrollador de la aplicación de la FAO en Roma, se tradujeron y utilizaron el cuestionario y la matriz para la evaluación de la resiliencia en 16 fincas agroecológicas situadas en los alrededores del Valle Central de Costa Rica. Una vez colectados e ingresados los datos en SHARP, se llevaron a cabo tres análisis: 1) comparación del perfil de las fincas estudiadas con indicadores a nivel nacional; 2) análisis de brechas para identificar las oportunidades de mejora en términos de resiliencia climática; 3) un análisis para adaptar la herramienta al contexto nacional. A pesar de que las fincas seleccionadas para el estudio son reconocidas como agroecológicas, su nivel medio de resiliencia medido $\left(\mathrm{x}^{-}=15,25 ; \mathrm{s}=1,16\right)$ sugiere la necesidad de implementar prácticas de manejo que contribuyan con la construcción de agroecosistemas más resilientes.
\end{abstract}

Palabras clave

Adaptación, agroecología, agroecosistemas, cambio climático, desarrollo rural. 


\begin{abstract}
Considering the current variability of climate, it is urgently required to measure and monitor climate resilience to inform and empower small agrosilvopastoral producers. Although there are other ways to measure resilience, the new SHARP tool developed by FAO allows the identification, measurement and prioritization of actions to improve climate resilience in a participatory manner, taking into account the interests of producers. In coordination with the FAO implementation team in Rome, the questionnaire and the matrix for resilience assessment were translated and used to assess 16 agroecological farms located within the Central Valley of Costa Rica. Once the data were collected and entered into SHARP, three analyses were carried out: 1) comparing the profile of the farms studied with indicators at a national level; 2) a gap analysis to identify opportunities for improvement in terms of climate resilience; 3 ) an analysis to adapt the tool to the national context. Although the farms selected for the study are recognized as agroecological, their average level of resilience $\left(x^{-}=15,25 ; s=1,16\right)$ suggests the need to implement management practices that contribute to the construction of more resilient ecosystems.
\end{abstract}

\title{
Keywords
}

Adaptation, agroecology, agroecosystems, climate change, rural development. 


\section{INTRODUCCIÓN}

El cambio climático global es uno de los mayores problemas ambientales a los que la sociedad actual debe hacer frente (1). Estimaciones sobre los gases de efecto invernadero (2) muestran que las emisiones procedentes de la agricultura, la silvicultura y la pesca se han elevado en los últimos 50 años, y podrían aumentar en un 30\% para el 2050 si no se realiza un esfuerzo mayor para reducirlas (3). La adaptación de los sistemas alimentarios al cambio climático es esencial para fomentar la seguridad alimentaria, la mitigación de la pobreza, la gestión sostenible y la conservación de los recursos naturales (2-5). Muchos países, incluyendo Costa Rica (6), ya están afrontando las repercusiones del cambio climático, conviviendo con una pluviometría irregular e impredecible, un aumento en la incidencia de tormentas y sequías prolongadas provocadas por lo que eran considerados eventos típicos como El Niño Oscilación Sur (ENOS), lo cual favorece la aparición de plagas y enfermedades que afectan personas, cultivos y animales (7).

La resiliencia, definida como la habilidad de un sistema para recobrarse, reorganizarse y evolucionar en respuesta a un estrés o disturbio externo (8-11) puede ser vista desde dos dimensiones: la resistencia a los eventos extremos y la capacidad de un sistema para mantener su estructura organizacional y su productividad tras una perturbación (12). Un agroecosistema es considerado ecológicamente resiliente si es capaz de mantener la productividad, a pesar de eventos extremos, siendo la biodiversidad clave para que los ecosistemas funcionen y provean servicios. No obstante, una mayor productividad y capacidad adaptativa del sistema ecológico no está estrictamente ligada con una mayor resiliencia social o reducción de pobreza de las personas que lo rodean (13).

Autores como Altieri y Nicholls (9) han postulado que los sistemas agroecológicos son más resilientes al cambio climático que los sistemas de producción agrícola convencional, ya que son más sensibles a las complejidades de la agricultura local. Dichos sistemas agroecológicos permiten abarcar la sustentabilidad, la soberanía alimentaria, la estabilidad biológica, la conservación de los recursos, la equidad de género, así como la productividad, con el fin de promover tecnologías de producción estables y de alta adaptabilidad ambiental $(13,14)$.

Los países necesitan conocimientos certeros y confiables sobre la vulnerabilidad de sus sistemas alimentarios, ecosistemas, sociedades y economías ante los efectos actuales y futuros del cambio climático; para esto se requiere entender no solamente el nivel de susceptibilidad y exposición a una amenaza, sino también su nivel de resiliencia (16). Sin embargo, medir la resiliencia climática no es fácil por ser un constructo multi-dimensional (17); es decir, requiere de la medición de múltiples indicadores ecológicos y socioeconómicos a un nivel más operativo para determinar su estado.

Aunque existen formas alternativas para medir resiliencia, la herramienta de Autoevaluación y Valoración Holística de la Resiliencia Climática de Agricultores y Pastores (SHARP, por sus siglas en inglés) permite la identificación, medición y priorización de acciones para mejorar la resiliencia climática de una manera participativa, tomando en cuenta los intereses de los productores. Su base teórica se sustenta en el marco de resiliencia agroecosistémica de Cabell y Oelofse (18), y en el aporte metodológico de Dixon y Stringer (19), en donde se toman en consideración los siguientes parámetros 
sobre el sistema productivo bajo evaluación: auto-organización social, auto-regulación ecológica, conexiones apropiadas, diversidad funcional, heterogeneidad espacial y temporal, integración con el capital natural local, autonomía global, mantenimiento de legados, óptima redundancia como medida de respaldo ante los disturbios expuestos, auto-reflexión y aprendizaje.

SHARP se elaboró entre el 2013 y el 2015 en consulta con más de 150 expertos. Fue probada en cerca de 450 fincas en nueve países africanos, en consulta en línea con académicos y desarrolladores de proyectos (20). De acuerdo con FAO (21), esta herramienta responde a la necesidad de comprender las circunstancias, preocupaciones e intereses con respecto a la resiliencia climática, a la vez que contribuye a la planificación y monitoreo de proyectos, mediante la identificación de áreas de baja resiliencia y del establecimiento de líneas de base sobre las cuales se pueden evaluar los cambios.

A partir de una autoevaluación de la resiliencia al cambio climático, SHARP permite a los agricultores priorizar acciones para construir resiliencia en sus sistemas productivos, y permite a las instituciones gubernamentales mejorar las políticas para fortalecer la resiliencia al clima cambiante.

En la presente investigación se tiene por objetivo evaluar el nivel de resiliencia al cambio climático de fincas agroecológicas situadas en los alrededores del Valle Central de Costa Rica utilizando la herramienta SHARP. Considerando que el manejo de la tierra en estas fincas es ambientalmente preferible, con un plan de acción basado en su capacidad productiva para mejorar la disponibilidad y calidad de los alimentos, así como por maximizar el uso de los recursos y energías del sistema de acuerdo con las características agroecológicas y socioeconómicas, se podrían esperar altos niveles de resiliencia en estas fincas (9).

\section{METODOLOGÍA}

Este estudio utilizó la herramienta SHARP de la FAO para la medición de la resiliencia al cambio climático en 16 fincas agroecológicas situadas en los alrededores del Valle Central de Costa Rica, según se detalla en la tabla 1. Estas fincas son reconocidas por sus características agroecológicas y socioeconómicas que buscan la sostenibilidad. La mayoría están identificadas por el Ministerio de Agricultura y Ganadería (MAG) como fincas integrales didácticas dentro del Programa de Fomento de la Producción Agropecuaria Sostenible.

En coordinación con el equipo desarrollador de la aplicación de la FAO en Roma, el cuestionario se transcribió del inglés para su posterior utilización en las fincas, al igual que la matriz para la evaluación de la resiliencia. Aunque la encuesta inicial contaba con 54 preguntas, se pudo adaptar al contexto nacional sin afectar el puntaje de resiliencia. Cada pregunta incluyó la evaluación de cuatro componentes: i) un componente estructurado con preguntas cerradas, normalmente, de escogencia única entre múltiples opciones (ej. ¿Utiliza usted nuevas variedades no nativas como variedades importadas, modificadas, de mayor rendimiento y/o producidas de manera privada? R./ Sí, No, No sé); ii) un componente no estructurado con preguntas abiertas donde se hacen 
Tabla 1. Ubicación, perfil climático y resiliencia de las fincas agroecológicas incluidas en el estudio y nivel de resiliencia estimado.

\begin{tabular}{cllccccc}
\hline ID & Cantón & Producto principal & $\begin{array}{c}\text { Biotemp. } \\
\left({ }^{\circ} \mathrm{C}\right)\end{array}$ & $\begin{array}{c}\text { Precip. } \\
(\mathrm{mm})\end{array}$ & $\begin{array}{c}\text { Altitud } \\
(\mathrm{m} . \mathrm{s} . n . m)\end{array}$ & $\begin{array}{c}\text { Zona de } \\
\text { Vida }\end{array}$ & $\begin{array}{c}\text { Resiliencia } \\
(\text { Escala 0-30) }\end{array}$ \\
\hline 1 & San Ramón & Raíces y frutas & $24-30$ & $4000-8000$ & 320 & bmh-T12 & 16,59 \\
2 & Zarcero & Hortalizas & $12-18$ & $2000-4000$ & 1760 & bmh-MB & 16,22 \\
3 & Zarcero & Café y hortalizas & $12-18$ & $1000-2000$ & 1712 & bh-MB & 16,35 \\
4 & Zarcero & Fresas & $12-18$ & $2000-4000$ & 2100 & bmh-MB & 14,08 \\
5 & Zarcero & Hortalizas & $12-18$ & $2000-4000$ & 1942 & bmh-MB & 16,79 \\
6 & Pacayas & Hortalizas & $12-18$ & $2000-4000$ & 2010 & bmh-MB & 13,45 \\
7 & Cartago & Hortalizas & $12-18$ & $1000-2000$ & 1750 & bh-MB & 15,49 \\
8 & Cartago & Hortalizas & $18-24$ & $1000-2000$ & 1150 & bh-P & 15,70 \\
9 & Moravia & Lácteos & $12-18$ & $2000-4000$ & 1400 & bmh-MB & 15,61 \\
10 & Coronado & Hortalizas & $12-18$ & $2000-4000$ & 1472 & bmh-MB & 16,36 \\
11 & Heredia & Hortalizas & $18-24$ & $2000-4000$ & 1508 & bmh-P & 14,50 \\
12 & Heredia & Hortalizas & $18-24$ & $2000-4000$ & 1414 & bmh-P & 15,46 \\
13 & Cartago & Hortalizas & $12-18$ & $1000-2000$ & 2000 & bh-MB & 15,85 \\
14 & Tarrazú & Café & $18-24$ & $2000-4000$ & 1500 & bmh-P & 14,64 \\
15 & La Unión & Hortalizas & $18-24$ & $2000-4000$ & 1400 & bmh-P & 13,79 \\
16 & Moravia & Hortalizas & $18-24$ & $2000-4000$ & 1394 & bmh-P & 13,20 \\
\hline
\end{tabular}

* bh-MB = Bosque húmedo montano bajo; bmh-MB = Bosque muy húmedo montano bajo; bh- $\mathrm{P}=$ Bosque húmedo premontano; bmh- $\mathrm{P}=$ Bosque muy húmedo premontano; bmh-T12 = Bosque muy húmedo tropical transición a montano.

observaciones particulares de acuerdo a cada finca (ej. Comente cuáles variedades en caso afirmativo o por qué no en caso negativo); iii) una evaluación propia por parte del agrosilvopastor de la satisfacción percibida respecto a qué tan adecuada es la característica bajo evaluación a la resiliencia de su sistema productivo (ej. ¿Qué tan adecuada es para su sistema productivo la combinación de especies nativas e introducidas? R./ Desde «nada adecuada» hasta «completamente adecuada» usando una escala Likert); iv) una evaluación por parte del agrosilvopastor sobre la importancia que pueda tener la característica bajo evaluación sobre su sistema productivo (ej. ¿Qué tan importante es para su sistema productivo la combinación de especies nativas e introducidas? R./ Desde «nada importante» hasta «completamente importante» usando una escala Likert). Los principales aspectos evaluados incluyeron: prácticas agrícolas, manejo pecuario, conservación de suelos, agua y energía, mejora genética, acceso a germoplasma, nivel de vida del productor y su familia, los cuales se circunscribieron dentro de las dimensiones productivas, económicas, ambientales, de interacción social y gobernanza. La aplicación de cada cuestionario requirió entre dos y cuatro horas por finca y se llevó a cabo en marzo del 2016. Adicionalmente, se georreferenció cada sistema productivo y se registró la altitud a la que se encontraba cada agroecosistema. Una vez colectada 
la información, esta se ingresó a la matriz de SHARP que utiliza Microsoft Excel para cuantificar el nivel de resiliencia, la cual está inversamente relaciona con el «ranquin de prioridad»; es decir, que entre menos resiliente una finca, esta debería atenderse con mayor prioridad. La resiliencia para cada aspecto evaluado se calcula utilizando la siguiente ecuación:

Resiliencia $=$ Puntaje del indicador + Grado de adecuación + Grado de importancia . donde:

- Puntaje de cada indicador: basado en el punto «i» mencionado anteriormente, el cual es comparado con el criterio de expertos sobre el grado de resiliencia, y normalizado a una escala de $0-10$.

- Grado de adecuación: resulta del punto «iii» mencionado anteriormente, y normalizado a una escala de 0-10.

- Grado de importancia: resulta del punto «iv» mencionado anteriormente, y normalizado a una escala «inversa» de 0-10 (a mayor importancia asignada menor valor en la escala).

Por ejemplo, si el acceso al crédito es inexistente (valor=0), es considerado completamente inadecuado por el productor (valor $=0$ ); a su vez, es considerado como algo muy importante para el bienestar del sistema o medio de vida (valor $=0$ ), entonces este indicador tendría un valor 0 de 30 , lo cual lo hace altamente prioritario. Los valores obtenidos permiten evaluar el nivel de resiliencia relativo de cada finca agroecológica (sistema productivo) y al mismo tiempo compararlas entre ellas de manera que permita examinar y prestar atención a las acciones prioritarias para hacer el sistema productivo más resiliente. Además del análisis sugerido por el modelo SHARP-FAO, se incluye una sección donde se contrasta la realidad de los sistemas productivos analizados con algunos indicadores del VI Censo Agropecuario Nacional (22), relacionados con tamaño de fincas, tenencia de tierra, y otros. Adicionalmente, se efectuó un análisis de brechas para identificar las oportunidades de mejora en términos de resiliencia para cada sistema productivo, y se determinaron cuáles fortalezas y debilidades posee la herramienta SHARP-FAO para ser aplicada con mayor precisión a la realidad costarricense. Finalmente, el reporte fue retroalimentado a través de una presentación que se hizo vía Skype con una miembro del equipo de SHARP-FAO.

\section{RESULTADOS Y DISCUSIÓN}

\subsection{Perfil de las fincas analizadas e indicadores nacionales}

De acuerdo con el reporte Estado de la Nación (23), el país enfrenta un gran desafío al carecer de un relevo generacional que asuma la dirección de la producción agropecuaria costarricense. Al 2014, solo un 27,2\% de los habitantes vivía en comunidades rurales y apenas el 10,5\% de la población económicamente activa laboraba en actividades relacionadas con agricultura, ganadería y pesca; además, el INEC (22), en el Censo de Población 2011, determinó que el promedio de edad de la población económicamente activa era de 43,2 años, pero la de las personas que trabajan en agricultura ganadería 
y pesca era de 48,1 años, con diferencias entre los trabajadores por rama de actividad (industria manufacturera 43,8, construcción 42,3, finanzas y seguros 38,5, administración y servicios de apoyo 40,4, entre otros). A su vez el INEC (22) determinó en el Censo Agropecuario 2014 que la edad promedio del productor nacional alcanzó los 53,9 años.

Este elevado promedio de edad de las personas productoras del país se deriva del aumento en el promedio de años de vida de la población (79 años) y, en particular, de la falta de deseo de los hijos de los productores para seguir los pasos de sus padres. En esta actitud pesa, según lo señala el Plan Estratégico del MAG 2015-2018, que los hijos(as) de los productores "sufren de absoluta invisibilidad de su trabajo y poca o nula remuneración laboral, sus derechos son poco reconocidos en el ámbito familiar debido a la cultura patriarcal y, en el ámbito de la ciudadanía rural, tienen pocas oportunidades de asociación y de participación, de toma de decisiones y de búsqueda de construcción de proyectos de vida" (24). Los jóvenes se alejan de la actividad agrícola si no ven una manera de desarrollar y mejorar su condición socioeconómica a corto y largo plazo.

Esta tendencia hacia el envejecimiento de los agricultores queda reflejada en la Fig. 1, donde se observa que, dentro de las fincas analizadas, la población menor de 15 años representa menos del 5\% del total. Aunque en el presente estudio la intención no fue comparar las fincas evaluadas con el total de fincas existentes en el país, es destacable que las fincas agroecológicas visitadas son cuatro veces más pequeñas si se comparan con la extensión promedio de las fincas a nivel nacional. Lo anterior es relevante si se toma en consideración que solo el $31 \%$ de las fincas agroecológicas incluidas en el estudio realizan algún tipo de actividad no agrícola (como turismo, capacitación, procesamiento del café o artesanía) que les genere ingresos adicionales a lo percibido por el usufructo de la tierra. Por tanto, el 69\% de los sistemas de producción analizados deben de generar una rentabilidad lo suficientemente alta como para proveer las necesidades básicas (alimentación, educación, recreación, salud, vestido y vivienda) de las familias que los trabajan, sin depender de insumos externos y maximizando su productividad/unidad de área.

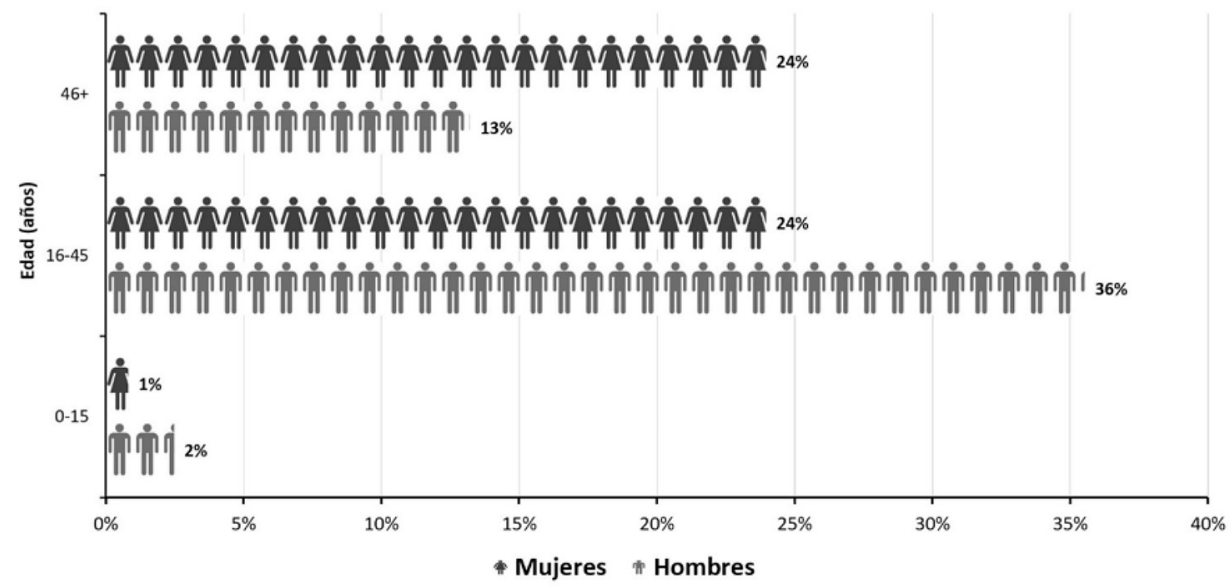

Figura 1. Composición del hogar por género y edad en las fincas evaluadas en marzo de 2016 (N=121). 
Un $25 \%$ de las fincas visitadas son dirigidas por mujeres (Tabla 2), mientras que a nivel nacional ellas encabezan el $15 \%$ del total de las unidades productivas. Aunque solo el $19 \%$ de las fincas en estudio se dedica a la agricultura y al pastoreo, cabe destacar que los productores han hecho un esfuerzo para incorporar el componente pecuario. Lo anterior es esencial en sistemas agroecológicos donde se busque reducir la dependencia de insumos externos, al tiempo que se favorece el ciclaje de nutrientes y energía dentro de la finca (9). En la Fig. 2 se hace referencia al tipo de actividades agrícolas complementarias que se realizan ordinariamente en las fincas, tal es el caso de la ganadería, la agroforestería, la acuacultura, la pesca y la avicultura. Todas ellas, si son desarrolladas apropiadamente, tienen un alto potencial de proveer servicios ecosistémicos e ingresos adicionales para los productores.

Tabla 2. Indicadores descriptivos de las fincas evaluadas y de fincas agropecuarias a nivel nacional.

\begin{tabular}{lcc}
\hline Indicadores & Fincas a nivel nacional* & Fincas evaluadas en el estudio \\
\hline Total de fincas & 93017 & 16 \\
Extensión promedio (ha/finca) & 25,9 & 6,6 \\
Tenencia simple de la tierra (\%) & 91,4 & 100 \\
Mujeres productoras (\%) & 15,6 & 25 \\
Fincas orgánicas $(\%)$ & 1,6 & 87,5 \\
\hline
\end{tabular}

* Elaborado con base en (22).

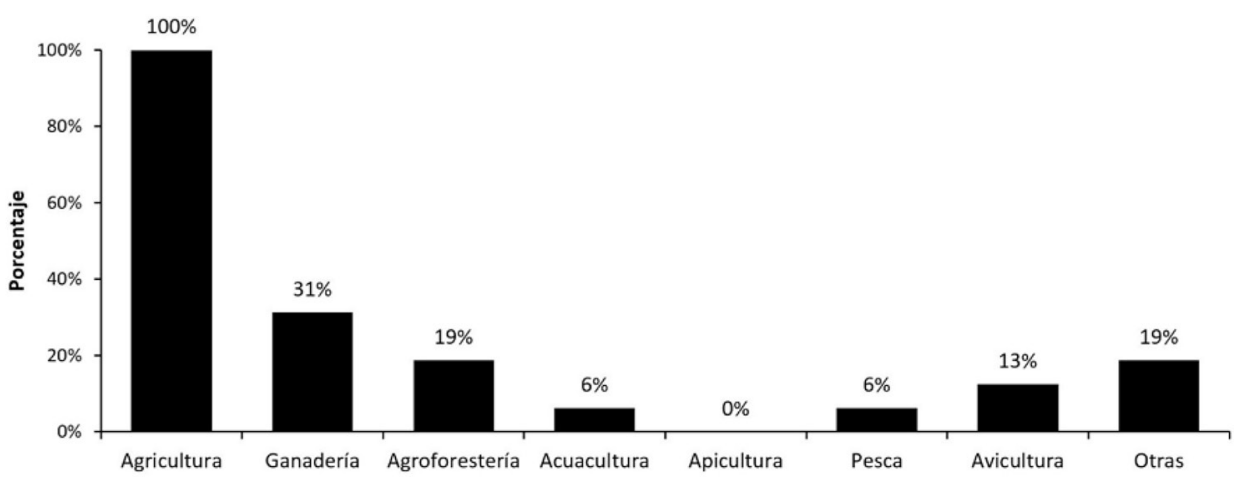

Figura 2. Actividades productivas llevadas a cabo en las fincas agroecológicas evaluadas en marzo de $2016(\mathrm{~N}=31)$.

Otra de las problemáticas que enfrenta el sector agropecuario nacional radica en las distorsiones del clima como consecuencia de la variabilidad en los patrones atmosféricos a nivel global. Si bien los agricultores agroecológicos costarricenses han visto 
la producción pecuaria como una aliada para diversificar y dar mayor valor agregado a su sistema productivo, es necesario invertir en la creación de capacidades que provean a estas fincas de una mayor posibilidad adaptativa para reducir su vulnerabilidad ante las principales distorsiones climáticas que los productores han identificado durante los últimos años, tal es el caso de los incrementos en la temperatura, la disminución o el aumento desmedido de las lluvias y los atrasos en el inicio de la estación lluviosa (Fig. 3). Independientemente del nivel de afectación de cada productor, es claro que la variabilidad en los patrones actuales del clima es cada vez mayor (23), y, por ende, se debe reducir la vulnerabilidad de las fincas ante esta condición partiendo no solamente del grado de exposición y resiliencia real (26), sino también a partir de las percepciones de los afectados que son una expresión de su realidad (27).

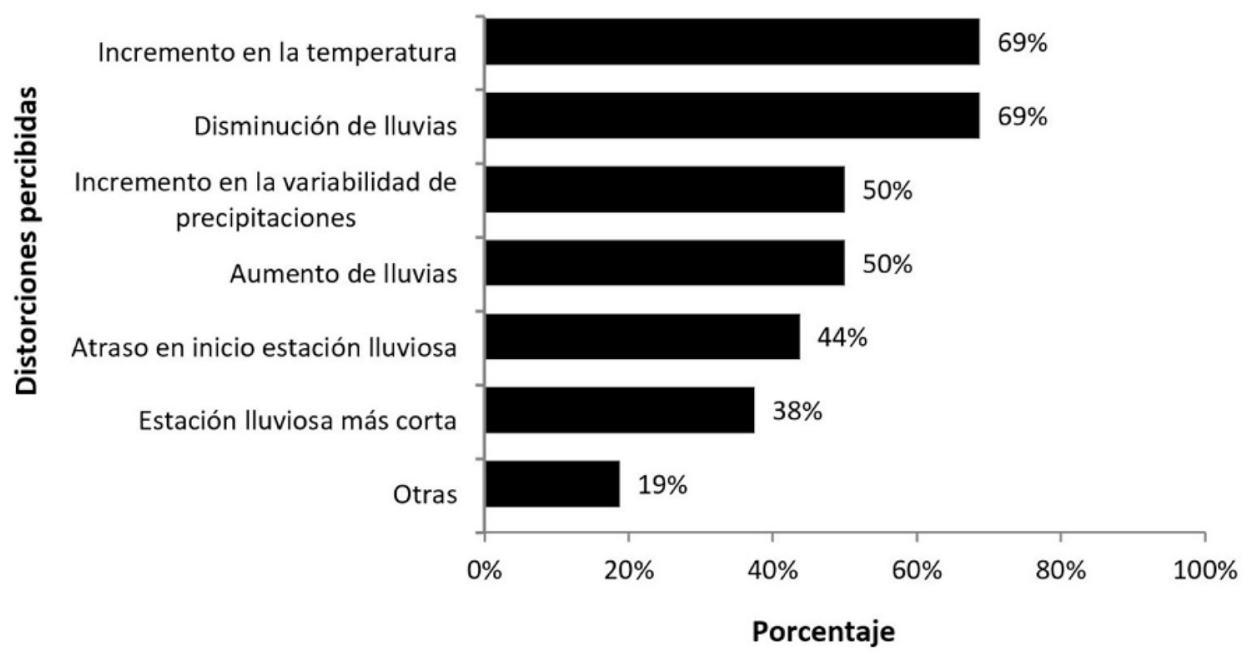

Figura 3. Principales distorsiones climáticas percibidas por los productores durante los cinco años previos a marzo $2016(\mathrm{~N}=54)$.

\subsection{Resultados de los indicadores de resiliencia}

Los valores de resiliencia presentaron un promedio de 15,25 puntos de 30 posibles, lo cual implica una resiliencia media de las fincas a pesar de su perfil agroecológico (Tabla 1). Es necesario hacer un análisis desglosado de los componentes de la medición de la resiliencia para comprender las fortalezas y debilidades de estas fincas estudiadas. Los valores de importancia promedios permiten visualizar dos grupos (Fig. 4). El primer grupo presenta indicadores con una alta importancia (valores entre 0 y 2,5). Estos indicadores muestran valores de resiliencia medios, a excepción de prácticas agrícolas y seguro de cosecha, con valores que tocaron el umbral de baja resiliencia (10 y 9 de 30 posibles puntos). La baja resiliencia en el indicador de seguros de cosecha se debe a que este parámetro presentó valores de adecuación muy bajos, ya que los productores consideran que las opciones de seguros de cosecha son muy limitadas o inexistentes. El segundo grupo lo componen indicadores con valores medios (valores mayores a 2,5 y menores a 8). Entre ellos están 
el uso de fertilizantes, acceso a infraestructura, actividades generadoras de ingresos no agrícolas, negociación directa con vendedores/consumidores, acceso a tierras, uso de prácticas de ahorro de energía, presencia de leguminosas, prácticas de manejo de arvenses, índice de diversidad de la dieta del hogar, y conocimiento de políticas de cambio climático y agricultura sostenible. Considerando el estado de las fincas y la menor calificación en la importancia que los productores asignaron a estos indicadores con respecto al primer grupo, los valores de resiliencia también se ubicaron en el umbral medio.

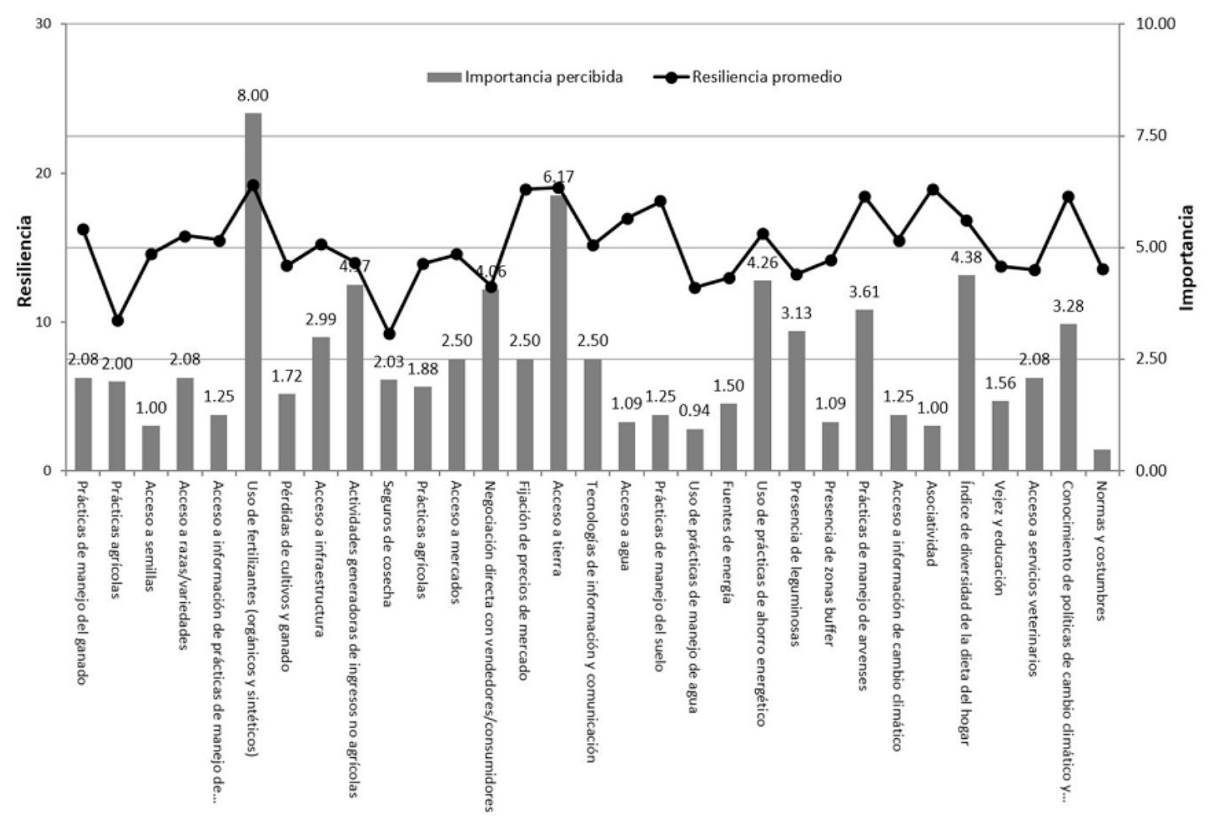

Figura 4. Evaluación del nivel medio de resiliencia e importancia percibida por los productorespara los indicadores contemplados en la herramienta SHARP.

Los indicadores de uso de fertilizantes, precio de mercado, acceso a tierra, prácticas en manejo de suelo, manejo de arvenses y asociatividad, obtuvieron las mayores calificaciones de resiliencia (cercanas a 19 puntos), mostrándose como las principales fortalezas de los sistemas agroecológicos que se desarrollan en las fincas estudiadas (Fig. 5). El uso de fertilizantes fue considerado por los productores como el indicador de menor importancia debido a que las propiedades de los suelos (químicas, físicas y biológicas) donde cultivan son buenas como consecuencia del aporte de materia orgánica realizado con los abonos orgánicos, lo que permite al sistema alcanzar una adecuada productividad sin necesidad de usar insumos externos como fertilizantes sintéticos, logrando resiliencia. Los precios de mercado, aunque fueron considerados por los productores como importantes debido a que su producción presenta una calidad diferenciada (la mayoría de ellos certificados orgánicos), pueden acceder a precios que consideran adecuados, por lo que se logra resiliencia en este aspecto. Las prácticas de manejo de suelo fueron consideradas de alta importancia; sin embargo, por las 
características de los agroecosistemas donde se implementaban diversas técnicas de conservación de suelos (28), los productores consideran adecuadas las prácticas que tienen en sus fincas y logran resiliencia. El manejo de arvenses es un indicador al cual los productores no le asignaron un valor alto de importancia, por tanto, el manejo es considerado adecuado; la combinación de estas propiedades permite a este indicador presentar uno de los valores de resiliencia más altos en la evaluación. Al indicador de acceso a la tierra, a pesar de que el terreno es esencial en los agroecosistemas, la importancia asignada por los productores fue de las más bajas, debido probablemente a que todos los encuestados tienen acceso al recurso sin que existan riesgos importantes de perder la tierra que cultivan. En el caso de la asociatividad, los productores le asignaron una importancia alta, pero por las facilidades de acceder a diferentes tipos de asociaciones, consideraron que la adecuación respecto a este indicador era alta y, por tanto, los sistemas logran resiliencia. A pesar de estos valores, se deben realizar esfuerzos para que lleguen al umbral de alta resiliencia.

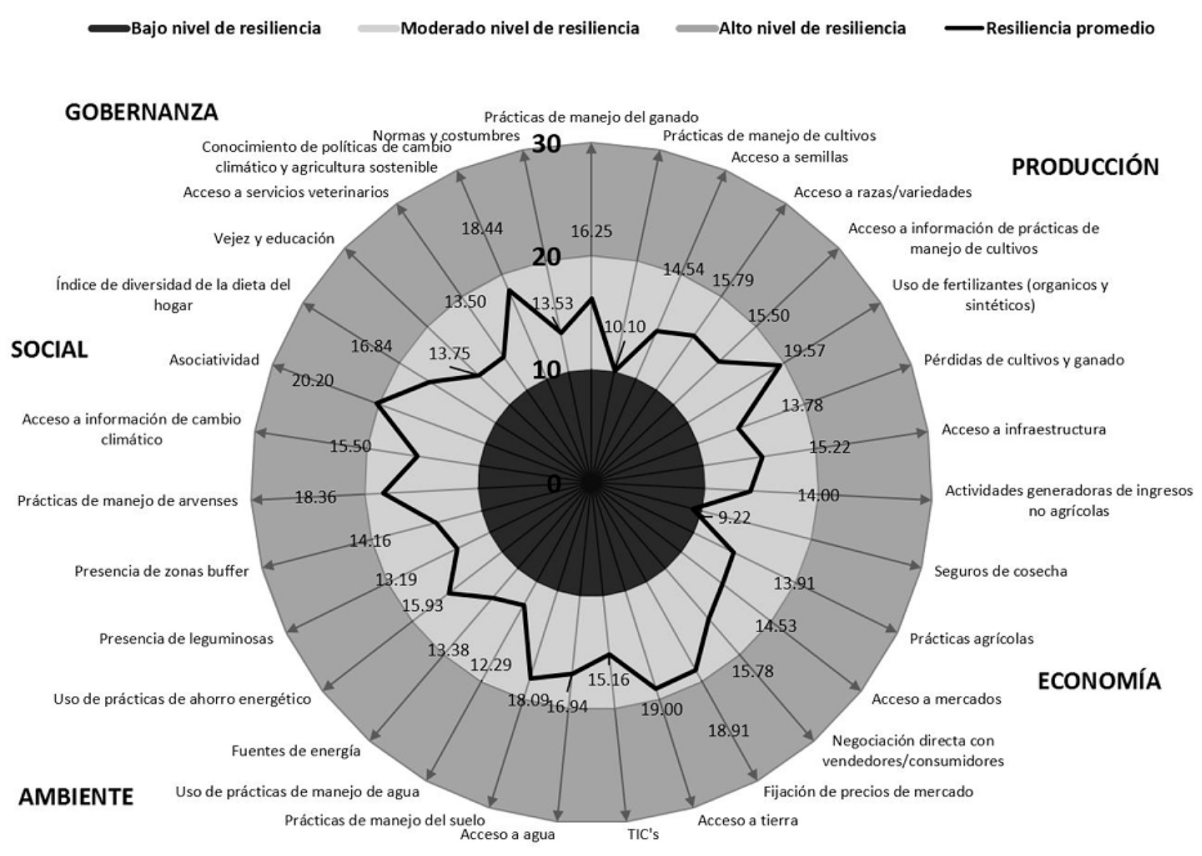

Figura 5. Nivel medio de resiliencia de las 16 fincas agroecológicas evaluadas utilizando la herramienta SHARP.

La adaptación del agroecosistema al clima se centra en la capacidad de recuperación de los ecosistemas agrícolas ante eventos climáticos hostiles (21). En la práctica esto implica que, frente a una perturbación de su sistema productivo, el productor recurre a los recursos que tiene a mano para tomar una acción de adaptación, tanto recursos propios (percepción, conocimiento, ubicación, experiencia) como externos (clima, suelo, estatus de las plagas, políticas gubernamentales y mercado); por consiguiente, 
la disponibilidad de estos recursos define su capacidad adaptativa local (29). Entonces, la resiliencia es una capacidad de recuperación que se puede fomentar por medio del aprendizaje, la flexibilidad, la participación y el empoderamiento $(8,20,27)$. Así pues, diversificar recursos y estrategias para cubrir las necesidades y los fines en cada uno de los indicadores permitiría potenciar la capacidad de recuperación ante eventos climáticos extremos y fomentar el empoderamiento del productor, generando una mayor resiliencia.

\subsection{Mejoras y adaptación de la herramienta a la realidad nacional}

Los sistemas naturales y sociales evolucionan de manera integral pero no se comportan linealmente (31). Por tanto, ajustar la herramienta SHARP es inevitable considerando que la realidad socioeconómica de las fincas donde se probó en Costa Rica es muy diferente a la de las fincas africanas a partir de las cuales se desarrolló la aplicación (21). Tal es el caso de las preguntas asociadas a la dieta familiar, al último tiempo de comida o a la tenencia y manejo de los ahorros familiares. En este sentido, se sugiere la incorporación de una escala temporal diferenciada para Costa Rica, ya que las familias campesinas no siempre producen para autoconsumo, sino que adquieren sus víveres con cierta frecuencia (semanal, quincenal o mensual). Además, la tenencia de ahorros está sujeta al pago de la cosecha y de las deudas en las que incurrieron durante el periodo productivo.

Las fincas evaluadas con SHARP se ubican en el Valle Central del país, que presenta diferencias culturales y climáticas con otras regiones más alejadas de la ciudad capital, por lo que es importante aplicar el instrumento a productores en otras zonas del país y cuyos resultados validen y amplíen las sugerencias de modificaciones a la aplicación producto de esta validación, y sea eficiente en la medida de la resiliencia de las fincas de Costa Rica.

Otro de los criterios que debe ajustarse al contexto nacional es el relacionado con el acceso a tierras comunales, ya que, a excepción de los territorios indígenas, donde este aspecto sería muy relevante, por lo general los productores nacionales cultivan en sus propias tierras o en terrenos alquilados. Las interrogantes asociadas a la toma de decisiones en el hogar también deberían de revisarse, ya que este aspecto es variable según la conformación actual de la familia costarricense y no aporta información tan crucial en términos de resiliencia social.

Durante la valoración de SHARP en las fincas, se determinó que la aplicación omite aspectos que podrían considerarse claves en el contexto de Costa Rica, como por ejemplo: tamaño de la finca, área cultivada, área de pastoreo y área forestal, personas con educación terciaria (universitaria o técnica), personas con discapacidad en la familia, existencia de trabajadores remunerados en la finca, así como también los seguros de salud y las pensiones para los miembros de la familia y los trabajadores. Estos aspectos son fundamentales para valorar capacidades colectivas que permitan amortiguar impactos negativos del clima, y para ayudar a impulsar nuevas estrategias de recuperación de la producción, e incluso de adaptación a la variabilidad climática y a sus consecuencias sobre los subsistemas que componen las fincas. 


\section{CONCLUSIONES}

Las fincas agroecológicas evaluadas dentro del estudio mostraron un nivel de resiliencia medio, con un alto desempeño en aspectos como uso de fertilizantes, asociatividad, tenencia de la tierra y fijación de precios. No obstante, se debe trabajar en el aseguramiento del relevo generacional para el sector agropecuario costarricense, lo que implica darles a los jóvenes mayores oportunidades para que vean la actividad agropecuaria como una opción de vida, y además, se debe buscar la mejora de indicadores como prácticas agrícolas, seguros de cosecha y en la adaptación de los indicadores de índole social a la realidad nacional.

Considerando la complejidad para estimar la resiliencia en las fincas, SHARP resultó ser una herramienta útil, práctica, y aplicable en el terreno, ya que permite realizar valoraciones técnicas y retroalimentar a los productores simultáneamente. Contar con una medición de resiliencia como esta para tecnificar la toma de decisiones a nivel de finca y para fortalecer la interacción propietario-extensionista es fundamental para aumentar la resiliencia de la producción agrosilvopastoril. Adicionalmente, se comprobó que los indicadores y la forma en que SHARP presenta los resultados del análisis son adecuados - con pequeños ajustes - para la realidad costarricense.

En el caso de Costa Rica, en donde se reconoce un importante avance en algunos aspectos de la agroproducción sostenible, como por ejemplo las Acciones de Mitigación Nacionalmente Apropiadas (NAMA por sus siglas en inglés) en café y ganado, resulta pertinente adaptar sus sistemas productivos a los cambios climáticos extremos cada vez más frecuentes. Por tanto, y dados los diferentes contextos físicos, biológicos, climáticos, y socioeconómicos que caracterizan las regiones productivas del país, se considera pertinente estimular la realización de estudios adicionales utilizando SHARP, que sistematicen las mejoras y se desarrolle una versión adaptada a la realidad nacional de SHARP, que aporten información para la toma de decisiones a nivel productivo y que contribuyan en la definición de políticas y programas que fortalezcan la resiliencia de sistemas agrosilvopastoriles tanto agroecológicos como convencionales para garantizar la calidad de vida de las familias de productores y la seguridad alimentaria del país.

\section{AGRADECIMIENTOS}

Agradecer a cada uno de los productores que nos abrieron las puertas en sus fincas agroecológicas para hacer este estudio. También quisiéramos agradecer a María Hernández Lagana y John Choptiany del equipo coordinador SHARP-FAO por su apertura y cooperación en este proyecto. Finalmente, agradecemos a la revista y las personas revisoras anónimas por sus atinadas observaciones.

\section{REFERENCIAS}

1. Córdoba Vargas CA, León Sicard TE. Resiliencia de sistemas agrícolas ecológicos y convencionales frente a la variabilidad climática en Anolaima (Cundinamarca - Colombia). Agroecología. 2013;8(1):21-32. 
2. FAO (Organización de las Naciones Unidas para la Alimentación y la Agricultura). Aumentan las emisiones de gases de efecto invernadero de la agricultura [Internet]. 2014. Disponible en: http://www.fao.org/news/story/es/item/218907/icode/

3. FAO (Organización de las Naciones Unidas para la Alimentación y la Agricultura). El estado de los bosques del mundo 2016 [Internet]. Rome: FAO; 2016. 138 p. Disponible en: http://www. fao.org/publications/sofo/2016/es/

4. Chandra A, Dargusch P, Mcnamara KE. How might adaptation to climate change by smallholder farming communities contribute to climate change mitigation outcomes? A case study from Timor-Leste, Southeast Asia. Sustain Sci. 2016;11(3):477-92.

5. Smith P, Olesen JE. Synergies between the mitigation of, and adaptation to, climate change in agriculture. J Agric Sci. 2010;148(5):543-52.

6. Flores Verdejo R, Salas Jiménez J, Astorga Molina M, Rivera Ugarte J. El impacto económico de los eventos naturales y antrópicos extremos en Costa Rica, 1988-2009. San José, Costa Rica: MIDEPLAN; 2010 p. 34.

7.Sequía en Centroamérica afecta a dos millones de personas. Diario La Prensa [Internet]. Disponible en: http://www.laprensa.hn/mundo/876017-410/ sequía-en-centroamérica-afecta-a-dos-millones-de-personas

8. Adger WN. Social and ecological resilience: are they related? Prog Hum Geogr. 2000 Sep 1;24(3):347-64.

9. Altieri MA, Nicholls CI. Agroecología y resiliencia al cambio climático: Principios y consideraciones metodológicas. Agroecología. 2013;8(1):7-20.

10. Carpenter S, Walker B, Anderies JM, Abel N. From Metaphor to Measurement: Resilience of What to What? Ecosystems. 2001 Dec 1;4(8):765-81.

11. Walker B, Holling CS, Carpenter S, Kinzig A. Resilience, Adaptability and Transformability in Social-ecological Systems. Ecol Soc [Internet]. 2004 Sep 16 [cited 2017 Jan 17];9(2). Disponible en: http://www.ecologyandsociety.org/vol9/iss2/art5/

12. Lin BB. Resilience in Agriculture through Crop Diversification: Adaptive Management for Environmental Change. BioScience. 2011 Mar 1;61(3):183-93.

13. Molina-Murillo S. Desarrollo verde e inclusivo en respuesta al cambio climático. Ambientico. 2016;258(Abril-Junio):24-9.

14. García A, Laurín M, Llosá MJ, Gonzálvez V, Sanz MJ, Porcuna JL. Contribución de la agricultura ecológica a la mitigación del cambio climático en comparación con la agricultura convencional. Agroecología. 2008 Apr 22;1(0):75-88.

15. Nicholls I, Leonardo R, Altieri MA, editors. Agroecología y resiliencia socioecológica: Adaptándose al cambio climático. Medellín, Colombia: REDAGRES-CYTED-SOCLA; 2013.207 p. 16. Watson R, Noble I, Bolin B, Ravindranath NH, Verardo D, Dokken D. Land Use, Land-Use Change and Forestry [Internet]. Cambridge, UK: IPCC; 2000: p. 375. Disponible en: http://www. ipcc.ch/ipccreports/sres/land_use/index.php?idp=0

17. Cumming GS, Barnes G, Perz S, Schmink M, Sieving KE, Southworth J, et al. An Exploratory Framework for the Empirical Measurement of Resilience. Ecosystems. 2005 Dec 1;8(8):975-87. 18. Cabell JF, Oelofse M. An Indicator Framework for Assessing Agroecosystem Resilience. Ecol Soc [Internet]. 2012;17(1). Disponible en: http://www.ecologyandsociety.org/vol17/iss1/art18/ 19. Dixon JL, Stringer LC. Towards a Theoretical Grounding of Climate Resilience Assessments for Smallholder Farming Systems in Sub-Saharan Africa. Resources. 2015 Mar 13;4(1):128-54. 20. Choptiany JMH, Phillips S, Graeub BE, Colozza D, Settle W, Herren B, et al. SHARP: integrating a traditional survey with participatory self-evaluation and learning for climate change resilience assessment. Clim Dev. 2016 May 14;8(5):1-13.

21. FAO (Organización de las Naciones Unidas para la Alimentación y la Agricultura). Autoevaluación y Valoración Holística de la Resiliencia Climática de Agricultores y 
Pastores (SHARP) [Internet]. 2015. Disponible en: http://www.fao.org/documents/card/en/c/ a78ba721-9e03-4cfc-b04b-c89d1a332e54/

22. Instituto Nacional de Estadística y Censos (INEC). Resultados Generales VI Censo Nacional Agropecuario [Internet]. San José, Costa Rica: Instituto Nacional de Estadística y Censos (INEC); 2015 p. 146. Disponible en: www.mag.go.cr/bibliotecavirtual/a00338.pdf

23. Programa Estado de la Nación. XXI Informe del Estado de la Nación en Desarrollo Humano Sostenible [Internet]. San José, Costa Rica: Programa Estado de la Nación; p. 431. Disponible en: http://estadonacion.or.cr/21/phone/index.html

24. MAG (Ministerio de Agricultura y Ganadería). Plan Estratégico 2015-2018. MAG; 2014. Disponible en: http://www.mag.go.cr/bibliotecavirtual/e14-10870.pdf

25. Retana J. Eventos hidrometeorológicos extremos lluviosos en Costa Rica desde la perspectiva de la adaptación al cambio en el clima. Rev Cienc Ambient. 2012 Dec 1;44(2):5-16.

26. Sánchez EM, Rojas AA, Rojas LG, Araya DR. Adaptación autónoma a eventos hidrometeorológicos extremos. Estudio de caso sobre la convivencia de un territorio indígena con el riesgo climático en la cuenca del río Sixaola, Costa Rica. Rev Cienc Ambient. 2016 Dec 5;50(2):20-37. 27. Molina Murillo SA. Urban Population Knowledge of Climate Change in Costa Rica and Nicaragua. Rev Latinoam Desarro Econ. 2013;19:55-75.

28. Gliessman S. Agroecología: procesos ecológicos en agricultura sostenible [Internet]. Turrialba, Costa Rica: CATIE; 2002. 359 p. Disponible en: https://docs.google.com/ file/d/0B26fmUn5W80zR2dsOFZISUh2YzA/edit

29. Comisión Europea. Estudio Temático 4: Inventario Buenas Prácticas Adaptación [Internet]. Programa EUROCLIMA, Dirección General de Desarrollo y Cooperación - EuropeAid, Comisión Europea; 2014 [cited 2017 Jan 30]. 114 p. Disponible en: http://euroclima.org/es/ servicios-de-informacion/libros-euroclima

30. Valerio-Hernández V, Arguedas-Quirós S, Aguilar-Arguedas A. Educación ambiental en el marco de una estrategia participativa para atender el cambio climático a nivel local: Experiencias en Costa Rica. Rev Cienc Ambient. 2016 Jun 24;49(2):1-12.

31. Folke C, Carpenter S, Elmqvist T, Gunderson L, Holling CS, Walker B. Resilience and Sustainable Development: Building Adaptive Capacity in a World of Transformations. AMBIO J Hum Environ. 2002 Aug;31(5):437-40. 